10. - Slinky models, Phys. Teach. 40 (2002) 324; available at http://dx.doi.org/10.1119/1. 1511579

11. M. Sawicki, Static elongation of a suspended Slinky ${ }^{\mathrm{TM}}$, Phys. Teach. 40 (2002) 276-278; available at http: //dx.doi.org/10.1119/1.1516379

12. T. P. Toepker, Center of mass of a suspended Slinky: an experiment, Phys. Teach. 42 (2004) 16-17; available at http://dx.doi.org/10.1119/1.1639962

\title{
An Intuitive Proof of the Singular Value Decomposition of a Matrix
}

Keith J. Coates (kcoates@ drury.edu) Drury University, Springfield MO

The Singular Value Decomposition (SVD) is very well known. We provide an intuitive proof for real matrices. The following statement is adapted from [1]:

Singular Value Decomposition Let the $m \times n$ real matrix A have rank $k$. Then there exist numbers $\sigma_{1} \geq \sigma_{2} \geq \cdots \geq \sigma_{k}>0$, called the singular values of $A$, an $m \times$ $m$ orthogonal matrix $U$ and an $n \times n$ orthogonal matrix $V$ such that $A=U \Sigma V^{T}$, where $\Sigma$ is the $m \times n$ matrix

$$
\Sigma=\left(\begin{array}{ll}
D & 0 \\
0 & 0
\end{array}\right)
$$

with $D$ the $k \times k$ diagonal matrix with diagonal element $d_{i i}=\sigma_{i}$ for $1 \leq i \leq k$.

The matrices $V$ and $\Sigma$ are constructed inductively as follows. With $v_{i}$ denoting the $i$ th column of $V$, we let $v_{1}$ be a unit vector in $\mathcal{R}^{n}$ such that $\left\|A v_{1}\right\|=\|A\|$, and for $2 \leq i \leq k$, we let $v_{i}$ be a unit vector in $\mathcal{R}^{n}$ such that

$$
\left\|A v_{i}\right\|=\max \left\{\|A v\|: v \in \mathcal{B}^{n} \cap\left(\operatorname{span}\left\{v_{1}, v_{2}, v_{3}, \ldots v_{i-1}\right\}\right)^{\perp}\right\},
$$

where $\mathcal{B}^{n}$ is the unit ball of $\mathcal{R}^{n}$ and $\|\cdot\|$ is the Euclidean norm or its induced matrix norm. The existence of each $v_{i}$ follows from the continuity of the norm and the compactness of closed subsets of $\mathcal{B}^{n}$. The set $\left\{v_{1}, v_{2}, v_{3} \ldots, v_{k}\right\}$ is obviously orthonormal. Setting $\sigma_{i}=\left\|A v_{i}\right\|$, we have $\sigma_{1} \geq \sigma_{2} \geq \cdots \geq \sigma_{k} \geq 0$. If $\sigma_{i}=0$ for some $i$, then $\sigma_{j}=0$ for all $j \geq i$ and the range of $A$ is spanned by $\left\{A v_{1}, A v_{2}, A v_{3}, \ldots, A v_{i-1}\right\}$, contradicting the fact that $A$ is of rank $k$. Thus $\sigma_{i}>0$ for $1 \leq i \leq k$. This constructs $D$ (and hence $\Sigma$ ), as well as the first $k$ columns of $V$.

We define $u_{i}$, the $i$ th column of $U$, to be $\frac{A v_{i}}{\sigma_{i}}$ for $1 \leq i \leq k$. Then $\left\|u_{i}\right\|=1$. The key question is, why does this inductive construction lead to the pairwise orthogonality of $\left\{u_{1}, u_{2}, u_{3}, \ldots, u_{k}\right\}$ ? The remaining $n-k$ columns of $V$ and $m-k$ columns of $U$ are easily constructed once we establish orthogonality. We use an approach that is quite simple, based on the trigonometric limit

$$
\lim _{\theta \rightarrow 0^{+}} \frac{1-\cos \theta}{\sin \theta}=0,
$$

which in turn implies that for any $\epsilon>0$, there exists $\delta>0$ such that

$$
\cos \theta+\epsilon \sin \theta>1, \text { for } 0<\theta<\delta .
$$

http://dx.doi.org/10.4169/college.math.j.42.5.394

MSC: 15A18, 15A23 
Since each induction step is a simple modification of the first, we show only the first step. That is, we start with $v_{1} \in \mathcal{B}^{n}$ such that $\left\|A v_{1}\right\|=\|A\|$ and show that for any $w$ nonzero in $\mathcal{R}^{n}$ with $w \perp v_{1}$, we have $A w \perp A v_{1}$. This implies that $u_{i} \perp u_{1}$ for $2 \leq i \leq k$.

Let $w \in \mathcal{R}^{n}$ satisfy $w \perp v_{1},\|w\|=1$, and $A w \neq 0$. If such a $w$ does not exist, then $k=1$ and the induction is finished. It is a basic result from linear algebra that there is a real number $\epsilon$ and a vector $u \in\left(\operatorname{span}\left\{A v_{1}\right\}\right)^{\perp} \subseteq \mathcal{R}^{m}$ such that $A w=\epsilon A v_{1}+u$. By replacing $w$ with $-w$, if necessary, we may assume $\epsilon \geq 0$. To show that $A w \perp A v_{1}$, we will show that $\epsilon=0$.

With $\theta$ acute and positive, let $y_{\theta}$ be the unit vector $(\cos \theta) v_{1}+(\sin \theta) w \in \mathcal{R}^{n}$. Then

$$
\begin{aligned}
A y_{\theta} & =(\cos \theta) A v_{1}+(\sin \theta) A w \\
& =(\cos \theta) A v_{1}+(\sin \theta)\left(\epsilon A v_{1}+u\right) \\
& =(\cos \theta+\epsilon \sin \theta) A v_{1}+(\sin \theta) u .
\end{aligned}
$$

The norm of $A y_{\theta}$ is at least as large as its component in the direction of $A v_{1}:\left\|A y_{\theta}\right\| \geq$ $(\cos \theta+\epsilon \sin \theta)\left\|A v_{1}\right\|$. But if $\epsilon$ is nonzero, by (2) we can choose $\theta>0$ small enough so that $(\cos \theta+\epsilon \sin \theta)\left\|A v_{1}\right\|>\left\|A v_{1}\right\|$. Then $y_{\theta}$ would be a unit vector and $\left\|A y_{\theta}\right\|>$ $\left\|A v_{1}\right\|=\|A\|$, a contradiction. We conclude that $\epsilon=0$ and $A w \perp A v_{1}$.

To complete the proof of the SVD, we point out that if $v \in\left(\operatorname{span}\left\{v_{1}, v_{2}, v_{3}, \ldots\right.\right.$, $\left.\left.v_{k}\right\}\right)^{\perp}$, then the above argument implies that $A v \in\left(\operatorname{span}\left\{u_{1}, u_{2}, u_{3}, \ldots, u_{k}\right\}\right)^{\perp}$. Since $A$ has rank $k$, this implies that $A v=0$. Otherwise, $\left\{u_{1}, u_{2}, u_{3}, \ldots, u_{k}, A v\right\}$ would be a linearly independent set in the range of $A$ with cardinality $k+1$. Thus we may choose the remaining $n-k$ columns of $V$ in any fashion that extends $\left\{v_{1}, v_{2}, v_{3}, \ldots, v_{k}\right\}$ to an orthonormal basis of $\mathcal{R}^{n}$, and likewise choose the remaining $m-k$ columns of $U$ to extend $\left\{u_{1}, u_{2}, u_{3}, \ldots, u_{k}\right\}$ to an orthonormal basis of $\mathcal{R}^{m}$. This completes the construction of the SVD of the matrix $A$.

Figure 1 is a visual depiction of the proof. Starting with the orthogonal unit vectors $v_{1}$ and $w$ and applying $A$ to the unit vector $y_{\theta}=(\cos \theta) v_{1}+(\sin \theta) w$ in $\mathcal{R}^{n}$, we have $A y_{\theta}=(\cos \theta) A v_{1}+(\sin \theta) A w$. If $A w$ were orthogonal to $A v_{1}$, then we could regard $A y_{\theta}$ as having "horizontal" vector component $(\cos \theta) A v_{1}$ and "vertical" component $(\sin \theta) A w$. But, under our assumption that $A w$ is not orthogonal to $A v_{1}$, we have $A w=\epsilon A v_{1}+u$, so that $(\sin \theta) A w=(\epsilon \sin \theta) A v_{1}+(\sin \theta) u$, with $\epsilon>0$ and $u \epsilon$ $\left(\operatorname{span}\left\{A v_{1}\right\}\right)^{\perp}$. The vector $(\sin \theta) u$ is now the vertical vector component of $A y_{\theta}$ and $(\epsilon \sin \theta) A v_{1}$ gets added to $(\cos \theta) A v_{1}$ to give us the horizontal component of $A y_{\theta}$. In the figure we see that as long as $\theta$ is small enough and nonzero, this extra piece added in the horizontal direction is enough to make the horizontal component of $A y_{\theta}$ longer than the length of $A v_{1}$, giving us our contradiction.

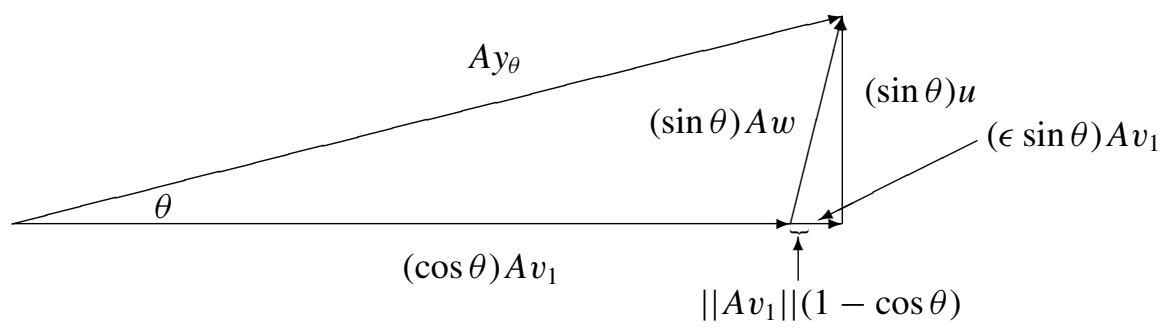

Figure 1. 
Summary. Using a simple trigonometric limit, we provide an intuitive geometric proof of the Singular Value Decomposition of an arbitrary matrix.

Acknowledgment. The author thanks Scott J. Simmons, a CMJ Classroom Capsule editor, the CMJ senior editor, and an anonymous referee for many helpful suggestions.

\section{References}

1. B. Noble and J. W. Daniel, Applied Linear Algebra, Prentice-Hall, Englewood Cliffs NJ, 1977.

\section{Discretization vs. Rounding Error in Euler's Method}

Carlos F. Borges (borges@ @ps.edu), Department of Applied Mathematics, Naval Postgraduate School, Monterey CA

Euler's method for solving initial value problems is a good vehicle for observing the relationship between discretization error and rounding error in numerical computation. As we reduce stepsize, in order to decrease discretization error, we necessarily increase the number of steps and introduce additional rounding error. The problem is common and can be quite troublesome. We will examine a simple device that can help delay the onset of this problem.

Meet the Eulers Consider the problem of solving the ordinary differential equation $\dot{x}=f(x, t)$ on the interval $[0, T]$, subject to the initial condition $x(0)=x_{0}$. A common technique for solving such a problem numerically is Euler's method. The method starts by selecting a positive integer $n$ and discretizing the time axis into a set of lattice points $t_{k}=k h$ for $k=0,1,2, \ldots, n$, where $h=T / n$ is called the step size. Provided that that $x(t)$ is sufficiently differentiable, we have, by Taylor's formula,

$$
x\left(t_{k}+h\right)=x\left(t_{k}\right)+h \dot{x}\left(t_{k}\right)+O\left(h^{2}\right) .
$$

Replacing $t_{k}+h$ by $t_{k+1}$ and $\dot{x}\left(t_{k}\right)$ by $f\left(x\left(t_{k}\right), t_{k}\right)$ yields

$$
x\left(t_{k+1}\right)=x\left(t_{k}\right)+h f\left(x\left(t_{k}\right), t_{k}\right)+O\left(h^{2}\right) .
$$

Ignoring the single-step discretization error $O\left(h^{2}\right)$ leads to the Euler approximation. Euler's method is the repeated use of this approximation across the entire lattice of discretized time points. In particular, we set $\psi_{0}=x_{0}$ and use the recurrence

$$
\psi_{k+1}=\psi_{k}+h f\left(\psi_{k}, t_{k}\right)
$$

for $k=0,1, \ldots, n-1$. If the above scheme is carried out in exact arithmetic, then we call $\psi_{n}$ the Euler solution and the difference $x(T)-\psi_{n}$ the discretization error for step-size $h$.

A less common derivation is to transform the initial value problem to the definite integral:

$$
x(T)=x_{0}+\int_{0}^{T} f(x, t) d t,
$$

http://dx.doi.org/10.4169/college.math.j.42.5.396

MSC: 65-01, 65-04, 65L05 